\title{
Strychnos L. da América do Sul e Central
}

\author{
M.A. Silva ${ }^{1,2}$, A.R.M. Souza-Brito ${ }^{4}$ C.A. Hiruma-Lima ${ }^{3}$, L.C.Santos ${ }^{1}$, M. Sannomiya ${ }^{1}$, \\ W. Vilegas ${ }^{1^{*}}$
}

${ }^{1}$ Departamento de Química Orgânica, Instituto de Química, UNESP, Caixa Postal 355, 14800-900, Araraquara, SP, Brasil,

${ }^{2}$ Departamento de Fármacos e Medicamentos, Faculdade de Ciências Farmacêuticas, UNESP, Caixa Postal 502, 14801-902, Araraquara, SP, Brasil,

${ }^{3}$ Departamento de Fisiologia, Instituto de Biociências, UNESP, Caixa Postal 510, Botucatu, 18618-000, SP, Brasil,

${ }^{4}$ Departamento de Fisiologia e Biofísica, Instituto de Biociências, UNICAMP, 13083-970, Campinas, SP, Brasil

\begin{abstract}
RESUMO: Uma revisão do perfil químico e farmacológico é apresentada de espécies de Strychnos (Loganiaceae) ocorrentes na América do Sul e Central, incluindo o uso popular, as substâncias isoladas e suas atividades biológicas.
\end{abstract}

Unitermos: Strychnos, Loganiaceae, alcalóides indólicos.

ABSTRACT: "Strychnos L. of South America and Central". A review of the chemical and pharmacological profile of Strychnos species (Loganiaceae) found in South and in Central America is presented. It includes the folk uses, the isolated compounds as well as the pharmacological activities as reported in the literature.

Keywords: Strychnos, Loganiaceae, indole alkaloids.

\section{INTRODUÇÃO}

O gênero Strychnos é constituído por 150 espécies, que ocorrem no mundo inteiro, especialmente nas Américas do Sul e Central, na África, na Ásia e na Austrália (Aimi et al., 1989). A grande maioria destas espécies ocorre na forma de cipós ou arbustos de pequeno porte (Lorenzi, 1998). Apenas S. pseudoquina, uma espécie nativa da América do Sul, se apresenta como uma árvore de médio porte, tendo a sua altura variando de 3 a $5 \mathrm{~m}$ de altura (Lorenzi, 1998).

Plantas do gênero Strychnos L. (Loganiaceae) são conhecidas desde há muito tempo devido às propriedades farmacológicas das substâncias presentes em algumas de suas espécies. Esses princípios em geral são tetanizantes nas espécies asiáticas e curarizantes nas espécies americanas (Ducke, 1965). Philippe (2004) menciona que as espécies americanas produzem alcalóides derivados da estricnina e, por isso, causam efeitos tetanizante; cita ainda que as espécies asiáticas produzem alcalóides, que causam os efeitos curarizantes. O curare é um veneno de origem vegetal preparado por algumas tribos de índios que vivem nas regiões montanhosas e cobertas de matas da América Equatorial. O curare tem aspecto de alcatrão, possui sabor muito amargo e pode ser produzido por dois grupos de plantas: das do gênero Chondrodendron (família Menispermaceae, $C$. tomentosum, C. toxicofera e C. candicans), cuja principal classe de alcalóides é a dos isoquinolínicos, e daquelas do gênero Strychnos (Loganiaceae), que produzem os alcalóides indólicos monoterpênicos (Bruneton, 1999). Pelas suas diversas propriedades tóxicas é usado pelos índios tanto na caça, quanto na guerra (Biocaa, 1954). Várias espécies de Strychnos são usadas na elaboração do curare, tendo ação tóxica, como por exemplo às espécies: S. amazonica, S. castelnaeana, S. divaricans, S. fendleri, S. froessi, S. gardneri, S. guianensis, S. macrophylla, S. melinoniana, S. mitscherlichii var mitscherlichii, S. mitscherlichii var amapensis, S. panamensis, S. parvifolia, S. solimoesana, S. subcordata, S. tomentas, S. trinervis e $S$. toxifera, esta última a mais potente dentre elas. Outras espécies são tóxicas para o sistema nervoso central, mas, surpreendentemente, a única espécie americana da qual foi isolada a estricnina é $S$. panamensis (Tabela 1).

Algumas espécies do gênero Strychnos têm importância comercial, sendo que a mais conhecida é $S$. ignatii empregada em homeopatia (Bruneton, 1999). O Escritório Europeu de Patentes já registrou o depósito de dezesseis pedidos, que envolvem desde o preparo de medicamentos contra insuficiência cardíaca e para o tratamento de dependência alcoólica até para a obtenção de surfactantes naturais (European, 2004).

Embora várias espécies sejam tóxicas, muitas vêm sendo utilizadas popularmente para diversas afecções. A maioria das espécies usadas popularmente emprega o chá das folhas ou cascas (Angenot et al., 
1990b; Frederich et al., 2000), com as subseqüentes ações farmacológicas: estimulante e tônica ( $S$. acuta, S. brasiliensis, S. guianensis, S.pseudoquina, $S$. rubiginosa e $S$. trinervis), contra problemas estomacais e abdominais (S. erichsonii, S. pseudoquina e S. trinervis), antipirética e antimalárica (S. brasiliensis, S. fendleri e $S$. pseudoquina), analgésica e antireumática (S. javariensis, S. panamensis e S. tansentosa), espasmolitica (S. toxifera), no tratamento de doenças venéreas (S. erichsonii), como abortiva (S. erichsonii), como afrodisíaca (S. erichsonii, S. melinoniana, S. mitscherlichii var. mitscherlichii, S. nigricans e $S$. oiapocensis) e no tratamento da anemia ( $S$. guianensis) (Tabela 1).

No levantamento bibliográfico realizado, utilizou-se, dentre outras, a base de dados Web of Science e o Medline, no período de 1970 até 2004. Esse levantamento mostra que foram investigadas 81 espécies americanas, o que representa 54\% do total das espécies descritas. As investigações fitoquímicas enfocam apenas o isolamento de alcalóides indólicos e bisindólicos (Tabela 1). Dos cerca de noventa alcalóides isolados, apenas um alcalóide bisbenzilisoquinolínico a warifiteina, foi relatado em S. divaricans (Mukherjee et al., 2003), (Tabela 1). Segundo Verpoorte (1977), os alcalóides indólicos monoterpênicos são subdivididos em 3 séries em função da substituição no $\mathrm{C}_{3}$ : a série normal apresenta um hidrogênio no $\mathrm{C}_{3}$, como por exemplo a estricnina (20); a série pseudo apresenta uma hidroxila no $\mathrm{C}_{3}$, como a 3-hidroxidiabolina (19b) e a série $\mathrm{N}$-metil-seco-pseudo apresenta uma carbonila no $\mathrm{C}_{3}$ e o nitrogênio apresentase substituído, geralmente com um grupamento metila, como a 10,11-dimetoxiestricnobrasilina (34b).

A literatura reporta algumas revisões sobre o gênero Strychnos (Bisset, 1970; Bisset et al., 1971; Bisset, 1974; Bisset et al., 1976; Ohiri et al., 1983), sendo que o mais recente data de 1990 (Angenot et al., 1990b). Nelas foram incluídas informações etnobotânicas, etnofarmacológicas e químicas de espécies ocorrentes em todo o mundo.

Dada a importância do gênero Strychnos, apresentamos uma revisão das espécies encontradas na América do Sul e Central, compreendendo o uso popular, as moléculas isoladas e suas atividades biológicas. As informações foram transcritas da forma como constam na literatura original, incluindo a forma como foram representadas as estruturas moleculares (Tabela 2). Alguns casos apenas relatam a ocorrência da espécie, sem qualquer investigação química ou farmacológica.

Os presentes autores estão investigando a constituição química e as ações farmacológicas das espécies $S$. pseudoquina, S. brasiliensis, S. bicolor e $S$. trinervis, dos estados do Tocantins e de São Paulo, onde os chás dessas espécies são usados contra afecções gástricas e no tratamento da malária.

\section{CONCLUSÃO}

Existem dois mecanismos tóxicos associados com os alcalóides presentes nas espécies de Strychnos: o efeito convulsivante - tetanizante, causado pela estricnina e seus derivados, e o efeito da paralisia dos músculos, efeito este causado pelos alcalóides quaternários presentes nas espécies (Philippe et al., 2004).

Em geral, pudemos observar que os alcalóides terciários estão envolvidos com a atividade tetanizante, enquanto que os alcalóides quaternários, particularmente os bis-indólicos, estão implicados na atividade curarizante. Isso pode ser explicado pela maior solubilidade dos alcalóides quaternários em água, e devido ao fato de que a atividade tetanizante é conseqüência de ação sobre o sistema nervoso central, ao passo que a ação curarizante se dá sobre as placas motoras que estão envolvidas na contração muscular.

As espécies da América do Sul e Central apresentam tanto os alcalóides quaternários quanto os derivados da estricnina. Aúnica espécie da América do Sul da qual foi isolada a estricnina é S. panamensis. Contudo, diversas outras espécies latino-americanas produzem derivados tóxicos da estricnina, o que alerta para o risco a que estão sujeitas as populações que usam essas espécies como fonte de cura para as suas enfermidades.

\section{AGRADECIMENTOS}

Os autores agradecem à Fapesp, pelo auxilio à pesquisa e pela bolsa concedida à M.S., ao $\mathrm{CNPq}$ , por bolsas à A.R.M.S.B. e W.V e à Capes, pela bolsa concedida a M.A.S. 
Tabela 1. Panorama químico-farmacológico de espécies do gênero Strychnos L. ocorrentes na América do Sul e Central.

\begin{tabular}{|c|c|c|c|}
\hline Espécies & Substâncias & $\begin{array}{c}\text { Atividade farmacológica } \\
\text { (* Uso popular) }\end{array}$ & Referências \\
\hline S. acuta & & *Estimulante e tônica. & Angenot et al., 1992. \\
\hline S. alvimiana & $\begin{array}{l}15 a, 15 b, 15 c, 15 d, 17 b, \\
43\end{array}$ & $\begin{array}{l}\text { 17b. Previne a resistência do } \\
\text { Plasmodiun falciparum a cloroquina }\end{array}$ & Angenot et al., 1992. \\
\hline S. amazonica & $\begin{array}{l}9 \mathrm{c}, 12 \mathrm{a}, 12 \mathrm{~b}, 14 \mathrm{a}, 18 \mathrm{a} \\
19 \mathrm{f}\end{array}$ & $\begin{array}{l}\text { 9c. Analgésica, hipotensora, } \\
\text { convulsivante. }\end{array}$ & Angenot et al., 1992. \\
\hline
\end{tabular}

S. arguaensis

S. asperula

S. atlantica

9b, 18a, 27

12a, 12b. Curarizante.

18a. Antimicrobiana, relaxante muscular, depressora do SNC, sedativa e antidiarréica.

18a. Antimicrobiana, relaxante muscular, depressora do SNC, sedativa e antidiarréica.

9b. Causa parada respiratória, convulsivante, hipotensora, sedativa. 27. Hipotensora, tranqüilizante.

S. bahiensis

S. barnhartiana

S. bicolor

S. brachiata

S. brachistantha

S. brasiliensis

S. bredemeyeri

S. castelnaeana

S. cayensis

S. cerradoensis

\section{S. chlorantha}

S. cogens

S. colombiensis

S. croatii

S. darienensis

S. davidsei

S. diaboli

S. divaricans

S. duckei

S. ecuadoriensis

S. erichsonii
$1 \mathbf{a}$

19a, 19f

15b, 15d, 16a, 16d, 17b, 17d, 17g, 33,

34a, 34b

19b, 19c, 19e, 38

19b, 19d

19a, 19b

12a, 12b, 14b, 18b, 31, 32, 36a, 37a, 41, 44

3, 10, 19a, 19b, 19d, 29 17b. Previne a resistência do Plasmodium falciparum à cloroquina. *Estimulante e tônica.

*Antimalárica

*Antipirética

19b. Convulsivante, causa parada respiratória, hipotensora, relaxante muscular.

38. Curarizante.

19b. Convulsivante, causa parada respiratória, hipotensora, relaxante muscular.

27. Hipotensora, tranqüilizante.

19b. Convulsivante, causa parada respiratória, hipotensora, relaxante muscular.

12a, 12b, 14b, 18b. Curarizante.

32. Espasmolítica.

36a, 37a. Curarizante.

19b. Convulsivante, causa parada respiratória, hipotensora, relaxante muscular.

* Usada para problemas estomacais e abdominais.

*Abortiva.

*Tratamento de doenças venéreas.

*Afrodisíaca.
Pinheiro, 2000.

Pinheiro, 2000.

Angenot et al., 1992.

Mukherjee et al.,

1997.

Oliveira et al., 1996

Angenot et al., 1992. Angenot et al., 1992. Angenot et al., 1992. Angenot et al., 1992. Angenot et al., 1992. Angenot et al., 1992. Iwataki et al., 1971.

Angenot et al., 1992. Angenot et al., 1992. Galeffi et al., 1982.

Pinheiro, 2000.

Pinheiro, 2000.

Angenot et al., 1992.

Angenot et al., 1992. Pinheiro, 2000.

Angenot et al., 1992. Angenot et al., 1992. Pinheiro, 2000.

Angenot et al., 1992.

Angenot et al., 1992.

Pinheiro, 2000.

Pinheiro, 2000.

Angenot et al., 1992.

Forgacs et al., 1986.

Juliem, 1999. 


\begin{tabular}{|c|c|c|c|}
\hline $\begin{array}{l}\text { S. eugeniifolia } \\
\text { S. fendleri }\end{array}$ & $\begin{array}{l}\text { 16a, 16b, 16e, 16f, 17a, } \\
17 e, 17 f, 19 b, 19 d\end{array}$ & $\begin{array}{l}\text { 19b. Convulsivante, causa parada } \\
\text { respiratória, hipotensora e relaxante } \\
\text { muscular. } \\
\text { 16e. Relaxante muscular. } \\
\text { *Antimalárica } \\
\text { *Antipirética }\end{array}$ & $\begin{array}{l}\text { Angenot et al., } 1992 . \\
\text { Angenot et al., } 1992 .\end{array}$ \\
\hline S. froessi & $\begin{array}{l}12 \mathrm{a}, 12 \mathrm{~b}, 14 \mathrm{a}, 18 \mathrm{~b}, 18 \mathrm{c} \\
19 \mathrm{a}, 19 \mathrm{~b} \\
36 \mathrm{a}, 36 \mathrm{~b}\end{array}$ & $\begin{array}{l}\mathbf{1 2 a}, \mathbf{1 2 b}, \mathbf{1 4 a}, \mathbf{1 8 b}, \mathbf{1 8 c} \text {. Curarizante. } \\
\text { 19b. Convulsivante, causa parada } \\
\text { respiratória, hipotensora e relaxante } \\
\text { muscular. } \\
\text { 36a, 36b. Curarizante. }\end{array}$ & Angenot et al., 1992. \\
\hline S. fulvotomentosa & & & Angenot et al., 1992. \\
\hline S. gardneri & $6,18 d, 19 f, 36 a$ & $\begin{array}{l}\text { 6. Convulsivante, citotóxica. } \\
\text { 18d, 36a. Curarizante. }\end{array}$ & $\begin{array}{l}\text { Angenot et al., } 1992 . \\
\text { Pinheiro, } 2000 .\end{array}$ \\
\hline S. glabra & 3 & & Angenot et al., 1992. \\
\hline S. goiasensis & & & Pinheiro, 2000. \\
\hline S. grayi & 3 & & Angenot et al., 1992. \\
\hline S. guianensis & $\begin{array}{l}1 \mathrm{~b}, 22 \mathrm{a}, 22 \mathrm{~b}, 23 \mathrm{a}, 23 \mathrm{~b} \\
23 \mathrm{c}, 24,25,26 \mathrm{a}, 26 \mathrm{~b} \\
36 \mathrm{a}\end{array}$ & $\begin{array}{l}\text { 1b. Inibe a monoamino oxidase, } \\
\text { citotóxica. } \\
\mathbf{2 2 b , 2 3 b . ~ A n t a g o n i s t a s ~ d o s ~ r e c e p t o r e s ~} \\
\text { colinérgicos nicotínicos. } \\
\text { 26a. Antagonista de receptores } \\
\text { colinérgicos. } \\
\text { 36a. Curarizante. } \\
\text { *Estimulante e tônica. } \\
\text { *Usada no tratamento da anemia. } \\
\text { *Inibe a transmissão neuromuscular. }\end{array}$ & $\begin{array}{l}\text { Angenot et al., } 1992 . \\
\text { Angenot et al., } 1995 . \\
\text { Manga et al., } 1996 . \\
\text { Penelle et al., } 1999 . \\
\text { Penelle et al., } 2000 . \\
\text { Penelle et al., } 2001 .\end{array}$ \\
\hline S. hirsuta & $5 a, 5 b$ & & $\begin{array}{l}\text { Angenot et al., } 1992 . \\
\text { Galeffi et al., } 1981 .\end{array}$ \\
\hline S. javariensis & 3 & $\begin{array}{l}\text { Analgésica } \\
\text { Antireumática }\end{array}$ & Angenot et al., 1992. \\
\hline S. jobertiana & $6,19 b, 19 c, 19 d$ & $\begin{array}{l}\text { 6. Convulsivante e citotóxica. } \\
\text { 19b. Convulsivante, causa parada } \\
\text { respiratória, hipotensora e relaxante } \\
\text { muscular. }\end{array}$ & Angenot et al., 1992. \\
\hline S. krukoffiana & & & Pinheiro, 2000. \\
\hline S. lobelioides & & & Pinheiro, 2000. \\
\hline S. macrophylla & $11,12 b, 12 d$ & 12a, 12b. Curarizante. & Angenot et al., 1992. \\
\hline S. malacasperma & $17 b, 17 c, 17 d, 17 h, 28$ & $\begin{array}{l}\text { 17b. Previne a resistência do } \\
\text { Plasmodiun falciparum a cloroquina. } \\
\text { 28. Antimitótica. }\end{array}$ & $\begin{array}{l}\text { Angenot et al., } 1992 . \\
\text { Anenot et al., } 1990 . \\
\text { Belem et al., } 2002 . \\
\text { Pinheiro, } 2000 .\end{array}$ \\
\hline S. medeola & $9 b, 19 f$ & $\begin{array}{l}\text { 9b. Causa parada respiratória, } \\
\text { convulsivante, hipotensora e sedativa. }\end{array}$ & Angenot et al., 1992. \\
\hline S. melinoniana & $\begin{array}{l}1 \mathrm{c}, 2,3,4,7,8,11,12 \mathrm{a}, \\
12 \mathrm{~b}\end{array}$ & $\begin{array}{l}\text { 1c. Antimitótica. } \\
\text { 12a, 12b, 14b. Curarizante. } \\
\text { *Afrodisíaca }\end{array}$ & Angenot et al., 1992. \\
\hline $\begin{array}{l}\text { S. mitscherlichii } \\
\text { v. mitscherlichii } \\
\text { S. mitscherlichii } \\
\text { v. pubescentior }\end{array}$ & $14 b, 37 a$ & 14b, 37a. Curarizante & $\begin{array}{l}\text { Angenot et al., } 1992 . \\
\text { Angenot et al., } 1992 .\end{array}$ \\
\hline $\begin{array}{l}\text { S. mitscherlichii } \\
\text { v. amapensis } \\
\text { S. neglecta }\end{array}$ & $\begin{array}{l}11,12 b, 36 a, 37 a, 37 c, \\
38\end{array}$ & 36a, 37a, 37c, 38. Curarizante. & $\begin{array}{l}\text { Angenot et al., } 1992 . \\
\text { Pinheiro, } 2000 .\end{array}$ \\
\hline
\end{tabular}




\begin{tabular}{|c|c|c|c|}
\hline S. nigricans & 3 & *Afrodisíaca & Angenot et al., 1992. \\
\hline S. oiapocensis & 3 & *Afrodisíaca & Angenot et al., 1992. \\
\hline S. pachycarpa & & & Pinheiros, 2000. \\
\hline S. panamensis & $\begin{array}{l}11,18 b, 19 b, 20,21,36 c, \\
37 c\end{array}$ & $\begin{array}{l}\mathbf{1 1}, \mathbf{1 8 b}, \mathbf{3 6 c}, \mathbf{3 7 c} \text {. Curarizante. } \\
\mathbf{1 9 b} \text {. Convulsivante, causa parada } \\
\text { respiratória, hipotensora e relaxante } \\
\text { muscular. } \\
\mathbf{2 0 , 2 1 . ~ C o n v u l s i v a n t e ~ e ~ r e l a x a n t e ~} \\
\text { muscular e provoca tétano muscular. } \\
\text { *Analgésica, antireumática. }\end{array}$ & Angenot et al., 1992. \\
\hline S. panurensis & & & Angenot et al., 1992. \\
\hline S. parviflora & 3 & & Angenot et al., 1992. \\
\hline S. parvifolia & $3,6,12 a, 12 b, 14 b, 37 a$ & $\begin{array}{l}\text { 6. Convulsivante e citótoxica. } \\
\text { 12a, } 12 b, 14 b, 37 a \text {. Curarizante. }\end{array}$ & Angenot et al., 1992. \\
\hline S. peckii & 3 & & Angenot et al., 1992. \\
\hline S. peoppigii & & & Angenot et al., 1992. \\
\hline S. progeliana & & & Pinheiros, 2000. \\
\hline S. pseudoquina & 18a, 19b, 19f. & $\begin{array}{l}\text { 18a. Antimicrobiana e relaxante } \\
\text { muscular, depressora do SNC, sedativa } \\
\text { e antidiarréica. } \\
\text { 19b. Convulsivante, causa parada } \\
\text { respiratória, hipotensora e relaxante } \\
\text { muscular. } \\
\text { *Estimulante e tônica. } \\
\text { *Usada para problemas estomacais e } \\
\text { abdominais. } \\
\text { *Antimalárica. } \\
\text { *Antipirética. }\end{array}$ & $\begin{array}{l}\text { Angenot et al., } 1992 . \\
\text { Nicoletii et al., } 1984 . \\
\text { Delle Monache et al., } \\
1969 .\end{array}$ \\
\hline S. pubiflora & & & Pinheiros, 2000. \\
\hline S. ramentifera & & & Angenot et al., 1992. \\
\hline S. recognita & & & Angenot et al., 1992. \\
\hline S. romeu-belenii & $19 f$ & & Angenot et al., 1992. \\
\hline S. rondeletioides & $19 a, 19 b$ & $\begin{array}{l}\text { 19b. Convulsivante, causa parada } \\
\text { respiratória, hipotensora e relaxante } \\
\text { muscular. }\end{array}$ & Angenot et al., 1992. \\
\hline S. rubiginosa & $9 b, 13,19 f$ & $\begin{array}{l}\text { 9b. Causa parada respiratória, } \\
\text { convulsivante, hipotensora e sedativa. } \\
\text { *Estimulante e tônica. }\end{array}$ & $\begin{array}{l}\text { Angenot et al., } 1992 . \\
\text { Bettolo et al., } 1980 .\end{array}$ \\
\hline S. sandwithiana & 19a & & Angenot et al., 1992. \\
\hline S. schultesiana & & & Angenot et al., 1992. \\
\hline S. shunkei & & & Pinheio, 2000. \\
\hline S. setosa & & & Pinheio, 2000. \\
\hline
\end{tabular}




\begin{tabular}{|c|c|c|c|}
\hline S. solerederi & $19 a, 19 b$ & $\begin{array}{l}\text { 19b. Convulsivante, causa parada } \\
\text { respiratória, hipotensora e relaxante } \\
\text { muscular. }\end{array}$ & Angenot et al., 1992. \\
\hline S. solimoesana & $\begin{array}{l}11,14 b, 19 b, 35,36 a \\
36 b, 36 c, 37 a, 37 b, 37 c \\
38\end{array}$ & $\begin{array}{l}\text { 11, 14b. Curarizante } \\
\text { 19b. Convulsivante, causa parada } \\
\text { respiratória, hipotensora e relaxante } \\
\text { muscular. } \\
\text { 36a, 36b, 36c. Curarizante. } \\
\text { 37a, 37b, 37c. Curarizante. } \\
\text { 38. Curarizante. }\end{array}$ & Angenot et al., 1992. \\
\hline S. subcordata & $11,12 \mathrm{a}, 12 \mathrm{~b}, 14 \mathrm{~b}, 19 \mathrm{a}$ & 11, 12a, 12b, 14b. Curarizante. & Angenot et al., 1992. \\
\hline S. tabascana & $15 a, 15 d, 16 c, 17 b, 17 c$ & $\begin{array}{l}\text { 16c. Relaxante muscular. } \\
\text { 17b. Previne a resistência do } \\
\text { Plasmodiun falciparum à cloroquina. }\end{array}$ & Angenot et al., 1992. \\
\hline S. tarapotensis & & & Angenot et al., 1992. \\
\hline S. tomentosa & $14 b, 18 c, 36 a, 36 b$ & 14b, 18c, 36a, 36b. Curarizante. & Angenot et al., 1992. \\
\hline S. toxifera & $\begin{array}{l}9 \mathrm{a}, 9 \mathrm{~b}, 9 \mathrm{c}, 9 \mathrm{~d} 11,12 \mathrm{a} \\
12 \mathrm{~b}, 18 \mathrm{a}, 18 \mathrm{~b}, 18 \mathrm{c}, 19 \mathrm{a}, \\
19 \mathrm{~d}, 19 \mathrm{~g}, 37 \mathrm{a} 7 \mathrm{~b}, 39 \mathrm{a} \\
39 \mathrm{~b}, 40\end{array}$ & $\begin{array}{l}\text { 9b. Causa parada respiratória, } \\
\text { convulsivante, hipotensora e sedativa. } \\
\text { 11, 12a, 12b. Curarizante. } \\
\text { 18a. Antimicrobiana e relaxante } \\
\text { muscular, depressora do SNC, sedativa } \\
\text { e antidiarréica. } \\
\text { 18b, 18c. Curarizante. } \\
\text { 37a, 37b, 39a, 39b. Curarizante. } \\
\text { 39b. Antimicrobiana e relaxante } \\
\text { muscular. } \\
\text { *Espasmolítica. }\end{array}$ & Angenot et al., 1992. \\
\hline S. trinervis & $\begin{array}{l}9 b, 14 b, 18 a, 18 d, 27,30 \\
36 a, 37 a, 42\end{array}$ & $\begin{array}{l}\text { 9b. Causa parada respiratória, } \\
\text { convulsivante, hipotensora e sedativa. } \\
\text { 14b. Curarizante. } \\
\text { 18a. Antimicrobiana e relaxante } \\
\text { muscular, depressora do SNC, sedativa } \\
\text { e antidiarréica. } \\
\text { 18d. Curarizante. } \\
\text { 27. Hipotensora e tranqüilizante. } \\
\text { 30. Espasmódica. } \\
\text { 36a, 37a. Curarizante. } \\
\text { *Estimulante e tônica. } \\
\text { *Previne problemas estomacais e } \\
\text { abdominais. } \\
\text { *Antimalárica. } \\
\text { *Antipirética. }\end{array}$ & $\begin{array}{l}\text { Angenot et al., } 1992 . \\
\text { Silva et al., } 1999 . \\
\text { Thomas et al., } 1992 . \\
\text { Demendeiros et al., } \\
1991 . \\
\text { Mukherjee et al., } \\
1990 .\end{array}$ \\
\hline
\end{tabular}

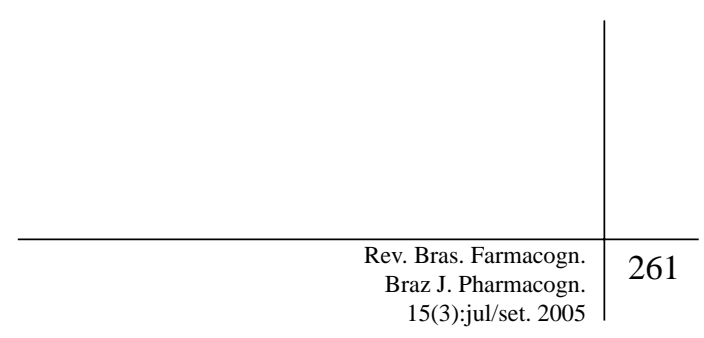


Tabela 2. Substâncias isoladas de espécies do gênero Strychnos L.

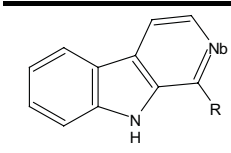

1a. norharmano $(\mathrm{R}=\mathrm{H})$

1b. harmano $\left(\mathrm{R}=\mathrm{CH}_{3}\right)$

1c. melinonina $\mathrm{F}\left(\mathrm{R}=\mathrm{CH}_{3}, \mathrm{~N}_{\mathrm{b}}=\mathrm{N}-\mathrm{CH}_{3}\right)$<smiles>C=Cc1cncc2c(=O)n3c(cc12)-c1[nH]c2ccccc2c1CC3</smiles>

3. angustina<smiles>COc1cccc2c1c1c3n2C2COC(C)C(CO)C2CPNC13</smiles>

5a. tetradesidroestricnohirsutina $\left(\Delta^{3(4), 5(6)}\right)$

5b. estricnohirsutina

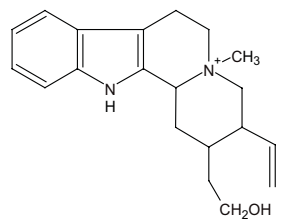

7. melinonina $\mathrm{B}$

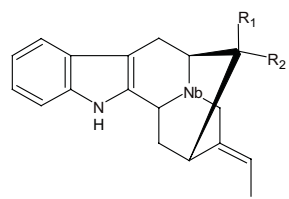

9a. mascusina $\mathrm{A}\left(\mathrm{R}_{1}=\mathrm{CH}_{2} \mathrm{OH}, \mathrm{R}_{2}=\mathrm{CO}_{2} \mathrm{CH}_{3}, \mathrm{~N}_{\mathrm{b}}=\mathrm{N}^{+}-\mathrm{CH}_{3}\right)$

9b. normascusina $\mathrm{B}\left(\mathrm{R}_{1}=\mathrm{H}, \mathrm{R}_{2}=\mathrm{CH}_{2} \mathrm{OH}, \mathrm{N}_{\mathrm{b}}=\mathrm{N}\right)$

9c. mascusina $\mathrm{B}\left(\mathrm{R}_{1}=\mathrm{H}, \mathrm{R}_{2}=\mathrm{CH}_{2} \mathrm{OH}, \mathrm{N}_{\mathrm{b}}=\mathrm{N}^{+}-\mathrm{CH}_{3}\right)$

9d. mascusina $\mathrm{C}\left(\mathrm{R}_{1}=\mathrm{COOCH}_{3}, \mathrm{R}_{2}=\mathrm{CH}_{2} \mathrm{OCH}_{3}\right.$,

$\mathrm{N}_{\mathrm{b}}=\mathrm{N}^{+}-\mathrm{CH}_{3}$ )

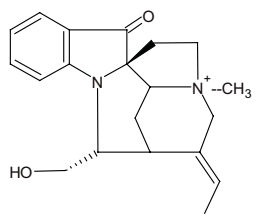

11. fluorocurina

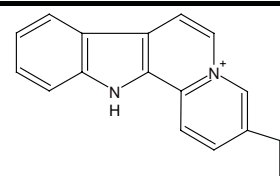

2. melinonina $G$ (flavopereirina)

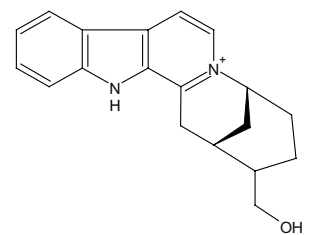

4. melinonina $\mathrm{E}$

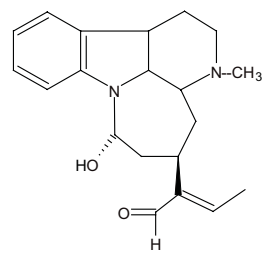

6. acagerina

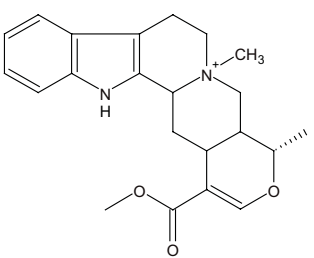

8. melinonina $\mathrm{A}$

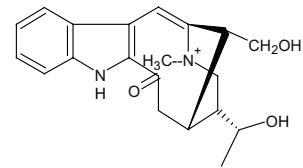

10. ericsonina

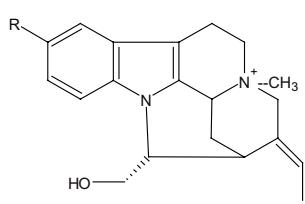

12a. mavacurina $B(R=H)$

12b. mavacurina $\mathrm{C}(\mathrm{R}=\mathrm{OH})$ 


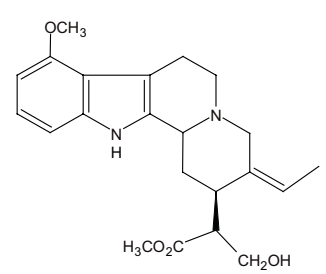

13. estricnorubigina

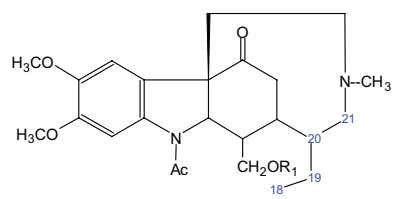

15a. tabascanina $\left(\mathrm{R}_{1}=\mathrm{H}, \Delta^{19(20)}\right)$

15b. estricnosilina $\left(R_{1}=A c, \Delta^{18(19), 20(21)}\right)$

15c. alvimina $\left(\mathrm{R}_{1}=\mathrm{Ac}, 19-\mathrm{OH}, \Delta^{20(21)}\right)$

15d. estricnosilidina $\left(R_{1}=A c, \Delta^{19(20)}\right)$

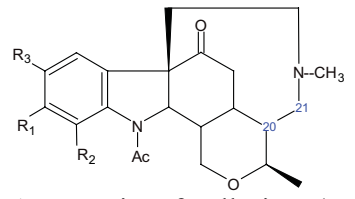

17a. estricnofendlerina $\left(R_{1}=R_{2}=R_{3}=H\right)$

17b. estricnobrasilina $\left(R_{1}=R_{2}=R_{3}=H, \Delta^{20(21)}\right)$

17c. 11-metoxiestricnobrasilina $\left(\mathrm{R}_{1}=\mathrm{OCH}_{3}, \mathrm{R}_{2}=\mathrm{H}\right.$,

$\left.\mathrm{R}_{3}=\mathrm{H}, \Delta^{20(21)}\right)$

17d. 12-hidroxi-11-metoxiestricnobrasilina

$\left(\mathrm{R}_{1}=\mathrm{OCH}_{3}, \mathrm{R}_{2}=\mathrm{OH}, \mathrm{R}_{3}=\mathrm{H}, \Delta^{20(21)}\right)$

17e. 12-hidroxi-11-metoxiestricnofendlerina $\left(\mathrm{R}_{1}=\mathrm{OCH}_{3}\right.$,

$\mathrm{R}_{2}=\mathrm{OH}, \mathrm{R}_{3}=\mathrm{H}$ )

17f. 11-metoxiestricnofendlerina $\left(\mathrm{R}_{1}=\mathrm{OCH}_{3}, \mathrm{R}_{2}=\mathrm{H}, \mathrm{R}_{3}=\mathrm{H}\right)$

17g. 10,11-dimetoxiestricnobasilina $\left(\mathrm{R}_{1}=\mathrm{OCH}_{3}, \mathrm{R}_{2}=\mathrm{H}\right.$,

$\left.\mathrm{R}_{3}=\mathrm{OCH}_{3}, \Delta^{20(21)}\right)$

17h. 12-hidroxi-10,11-dimetoxiestricnobrasilina

$\left(\mathrm{R}_{1}=\mathrm{OCH}_{3}, \mathrm{R}_{2}=\mathrm{OH}, \mathrm{R}_{3}=\mathrm{OCH}_{3}, \Delta^{20(21)}\right)$

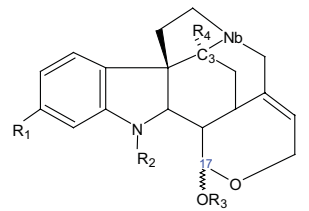

19a. caracurina VIII ( $N$-diacetildiabolina)

$\left(\mathrm{R}_{1}=\mathrm{R}_{2}=\mathrm{R}_{3}=\mathrm{R}_{4}=\mathrm{H}, 17-\mathrm{OH} \beta, \mathrm{N}_{\mathrm{b}}=\mathrm{N}\right)$

19b. diabolina $\left(R_{1}=R_{3}=R_{4}=H, R_{2}=A c, 17-O H \beta\right.$,

$\mathrm{N}_{\mathrm{b}}=\mathrm{N}$ )

19c. jobertina $\left(R_{1}=R_{4}=H, R_{2}=R_{3}=A c, 17-\right.$ OAc $\alpha$,

$\mathrm{N}_{\mathrm{b}}=\mathrm{N}$ )

19d. heningsamina $\left(R_{1}=R_{4}=H, R_{2}=R_{3}=\right.$ Ac, $17-$ OAc $\beta$, $\mathrm{N}_{\mathrm{b}}=\mathrm{N}$ )

19e. 3-hidroxidiabolina $\left(R_{1}=R_{3}=H, R_{2}=A c, R_{4}=O H\right.$,

$\mathrm{N}_{\mathrm{b}}=\mathrm{N}$ )

19f. 11-metoxidiabolina $\left(\mathrm{R}_{1}=\mathrm{OCH}_{3}, \mathrm{R}_{2}=\mathrm{Ac}, \mathrm{R}_{3}=\mathrm{OH}\right.$,

$\mathrm{R}_{4}=\mathrm{H}, \mathrm{N}_{\mathrm{b}}=\mathrm{N}$ )

19g. hemitoxiferina $\left(\mathrm{R}_{1}=\mathrm{R}_{2}=\mathrm{R}_{3}=\mathrm{R}_{4}=\mathrm{H}, 17-\mathrm{OH} \beta\right.$, $\mathrm{N}_{\mathrm{b}}=\mathrm{N}^{+}-\mathrm{CH}_{3}$ )

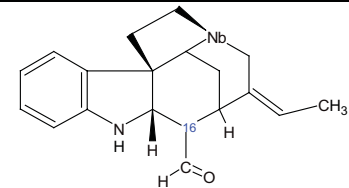

14a. nordiidrofluorocurarina $\left(\mathrm{N}_{\mathrm{b}}=\mathrm{N}\right)$

14b. fluorocurarina $\left(\mathrm{N}_{\mathrm{b}}=\mathrm{N}-\mathrm{CH}_{3}, \Delta^{2(16)}\right)$

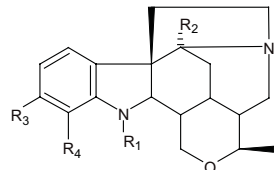

16a. espermoestricnina $\left(R_{1}=A c, R_{2}=H, R_{3}=H\right.$,

$\mathrm{R}_{4}=\mathrm{H}$ )

16b. estricnosplendina $\left(R_{1}=H, R_{2}=O H, R_{3}=H, R_{4}\right.$ $=\mathrm{H})$

16c. $\mathrm{N}$ - acetil - $\mathrm{O}$-metoxiestricnosplendina

$\left(\mathrm{R}_{1}=\mathrm{Ac}, \mathrm{R}_{2}=\mathrm{OCH}_{3}, \mathrm{R}_{3}=\mathrm{H}, \mathrm{R}_{4}=\mathrm{H}\right)$

16d. 12-hidroxi-11-metoxiespermoestricnina

$\left(\mathrm{R}_{1}=\mathrm{Ac}, \mathrm{R}_{2}=\mathrm{H}, \mathrm{R}_{3}=\mathrm{OCH}_{3}, \mathrm{R}_{4}=\mathrm{OH}\right)$

16e. $N$-acetilestricnoesplendina

$\left(\mathrm{R}_{1}=\mathrm{Ac}, \mathrm{R}_{2}=\mathrm{OH}, \mathrm{R}_{3}=\mathrm{H}, \mathrm{R}_{4}=\mathrm{H}\right)$

16f. $N$-acetil-12-hidroxi-11-metoxiestricnoespledina

$\left(\mathrm{R}_{1}=\mathrm{Ac}, \mathrm{R}_{2}=\mathrm{OH}, \mathrm{R}_{3}=\mathrm{OCH}_{3}, \mathrm{R}_{4}=\mathrm{OH}\right)$

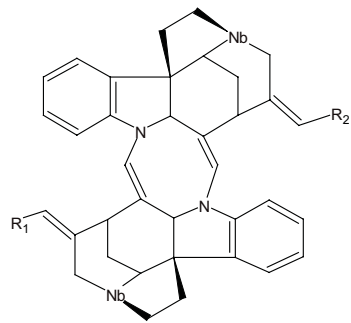

18a. bisnordiidrotoxiferina $\left(\mathrm{R}_{1}=\mathrm{R}_{2}=\mathrm{CH}_{3}, \mathrm{~N}_{\mathrm{b}}=\mathrm{N}\right)$

18b. diidrotoxiferina (Alcalóide $\mathrm{K}$ )

$\left(\mathrm{R}_{1}=\mathrm{R}_{2}=\mathrm{CH}_{3}, \mathrm{~N}_{\mathrm{b}}=\mathrm{N}^{+}--\mathrm{CH}_{3}\right)$

18c. toxiferina $\mathrm{I}\left(\mathrm{R}_{1}=\mathrm{R}_{2}=\mathrm{CH}_{2} \mathrm{OH}, \mathrm{N}_{\mathrm{b}}=\mathrm{N}^{+}--\mathrm{CH}_{3}\right)$

18d. C-alcaloide $\mathrm{H}\left(\mathrm{R}_{1}=\mathrm{CH}_{2} \mathrm{OH}, \mathrm{R}_{2}=\mathrm{H}, \mathrm{N}_{\mathrm{b}}=\mathrm{N}^{+}\right.$-

$\left.-\mathrm{CH}_{3}\right)$

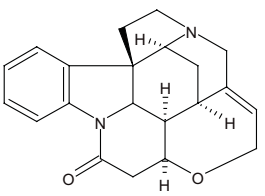

20. estricnina

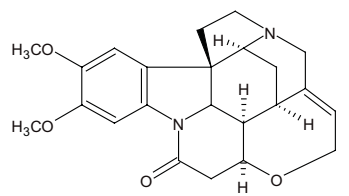

21. brucina 
M.A. Silva, A.R.M. Souza-Brito, C.A. Hiruma-Lima, L.C. Santos , M. Sannomiya , W. Vilegas

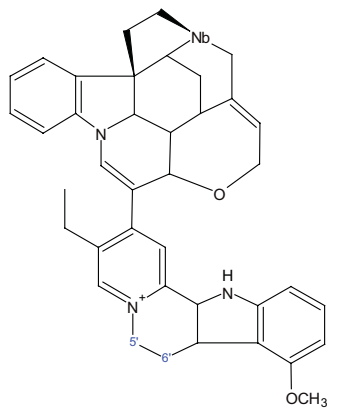

22a. 5',6'- deidroguiaflavina $\left(\mathrm{N}_{\mathrm{b}}=\mathrm{N}^{+}-\mathrm{CH}_{3}, \Delta^{5^{\prime}\left(6^{\prime}\right)}\right)$

22b. guiaflavina $\left(\mathrm{N}_{\mathrm{b}}=\mathrm{N}^{+}-\mathrm{CH}_{3}\right)$

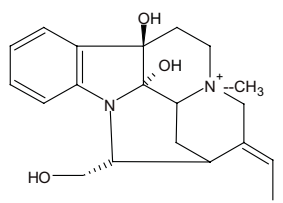

24. C-proflurocurina

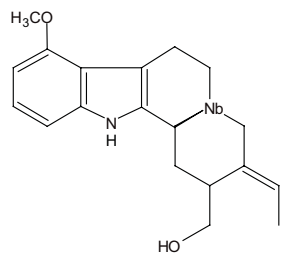

26a. 9-metoxi- $N$ - metilgeisoscizol $\left(\mathrm{N}_{\mathrm{b}}=\mathrm{N}^{+}-\mathrm{CH}_{3}\right)$

26b. metoxigeisoscizol $\left(\mathrm{N}_{\mathrm{b}}=\mathrm{N}\right)$

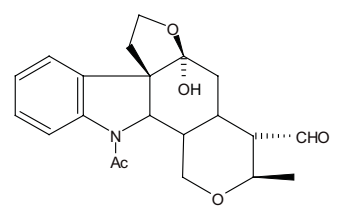

28. matogrosina

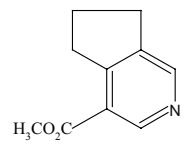

30. cantleina

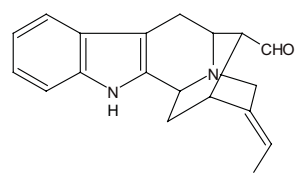

32. velosimina

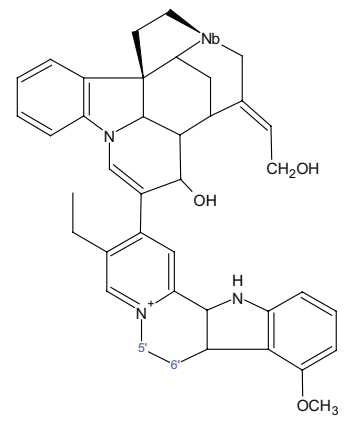

23a. 5,6' diidroguiacrisina $\left(\mathrm{N}_{\mathrm{b}}=\mathrm{N}^{+}-\mathrm{CH}_{3}, \Delta^{5^{\prime}\left(6^{\prime}\right)}\right)$

23b. guiacrisina $\left(\mathrm{N}_{\mathrm{b}}=\mathrm{N}^{+}-\mathrm{CH}_{3}\right)$

23c. guianensina $\left(\mathrm{N}_{\mathrm{b}}=\mathrm{N}, \Delta^{5^{\prime}\left(6^{\prime}\right)}\right)$

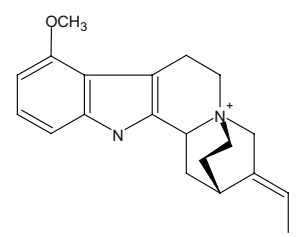

25. C - alcalóide O

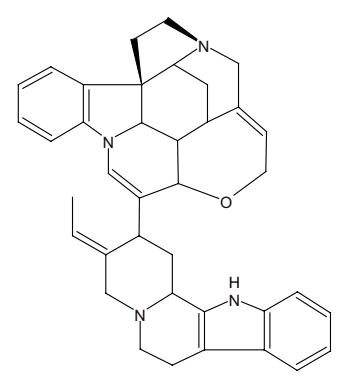

27. longicaudatina

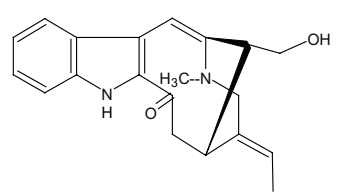

29. epiafinina

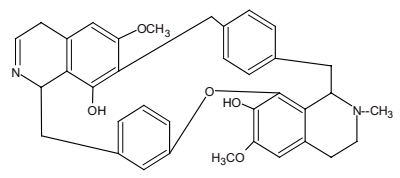

31. warifiteina

264

Rev. Bras. Farmacogn. Braz J. Pharmacogn. 15(3):jul/set. 2005 


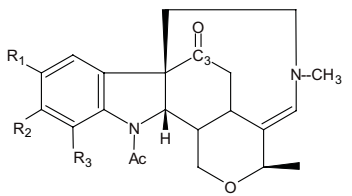

34a. 12-hidroxi-11-metoxiestricnobrasilina

$\left(\mathrm{R}_{1}=\mathrm{H}, \mathrm{R}_{2}=\mathrm{OCH}_{3}, \mathrm{R}_{3}=\mathrm{OH}\right)$

34b. 10,11-dimetoxiestricnobrasilina

$\left(\mathrm{R}_{1}=\mathrm{R}_{2}=\mathrm{OCH}_{3}, \mathrm{R}_{3}=\mathrm{H}\right)$

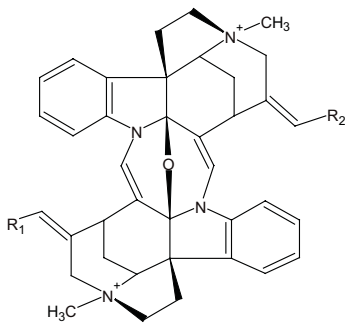

36a. $\mathrm{C}$ - curarina $\mathrm{I}\left(\mathrm{R}_{1}=\mathrm{R}_{2}=\mathrm{CH}_{3}\right)$

36b. alcalóide $\mathrm{E}\left(\mathrm{R}_{1}=\mathrm{R}_{2}=\mathrm{CH}_{2} \mathrm{OH}\right)$

36c. $\mathrm{C}$ - alcalóide $\mathrm{G}\left(\mathrm{R}_{1}=\mathrm{CH}_{3}, \mathrm{R}_{2}=\mathrm{CH}_{2} \mathrm{OH}\right)$

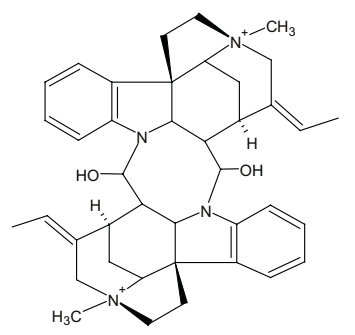

38. C - alcalóide D

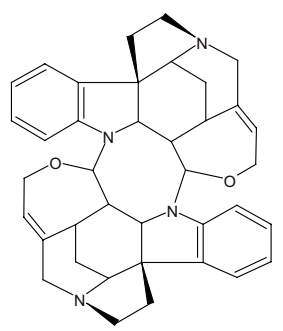

40. caracurina

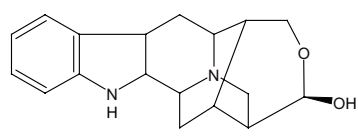

42. trinervina

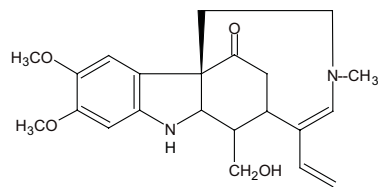

33. diacetilestricnosilidina

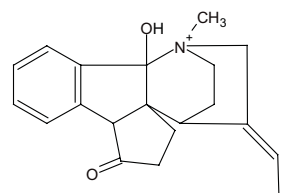

35. C- calebassinina

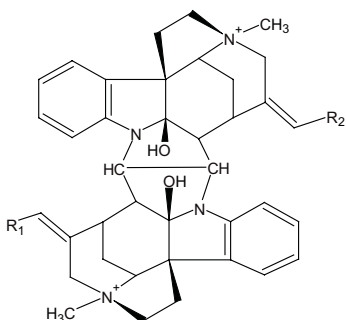

37a. $\mathrm{C}$ - toxiferina II $\left(\mathrm{R}_{1}=\mathrm{R}_{2}=\mathrm{CH}_{3}\right)$

37b. $\mathrm{C}$ - alcalóide $\mathrm{A}\left(\mathrm{R}_{1}=\mathrm{R}_{2}=\mathrm{CH}_{2} \mathrm{OH}\right)$

37c. $\mathrm{C}$ - alcalóide $\mathrm{F}\left(\mathrm{R}_{1}=\mathrm{CH}_{3}, \mathrm{R}_{2}=\mathrm{CH}_{2} \mathrm{OH}\right)$

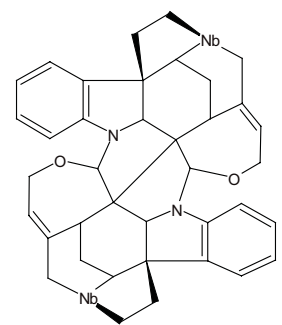

39a. toxiferina IX $\left(\mathrm{N}_{\mathrm{b}}=\mathrm{N}^{+}-\mathrm{CH}_{3}\right)$

39b. curacurina II $\left(\mathrm{N}_{\mathrm{b}}=\mathrm{N}\right)$

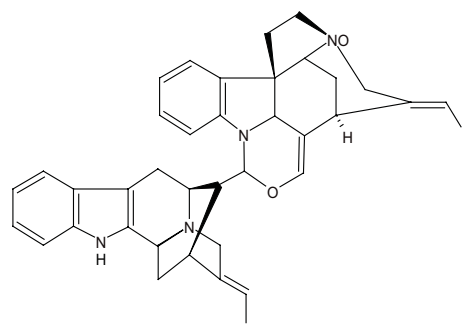

41. divaricina

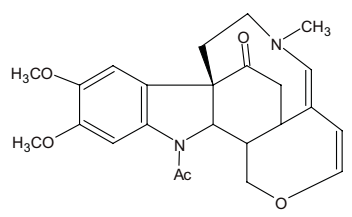

43. alviminina 


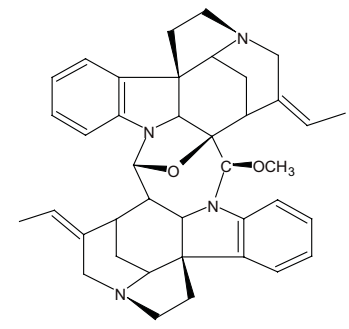

44. divarina

\section{REFERÊNCIAS}

Aimi N, Sakai SI, Ban Y 1989. Alkaloids of Strychnos and Gardneria species. In: The Alkaloids. California: Academic Press, INC, p. 1-69.

Angenot L, Belem PML, Imbiriba RAF, Poukens RP, QuetimLeclercq J, Warin R 1990a. An indolinic cryptoalkaloid from Strychnos mattogrossensis. Phytochemistry 29: 2746-2749.

Angenot L, Quertin-Leclercq J, Bisset NG 1990b. South american Strychnos species. Ethnobotany (except curare) and alkaloid screening. J Ethnopharmacol 28: $1-52$.

Angenot L, Quetin-Leclercq J, Llabres G, Warins R, Belem PML, Manga MH 1995. Guianensine, a zwitterionic alkaloid from Strychnos guianensis. Phytochemistry 40: 1557-1560.

Belem PML, Couceiro RPC, Rocha AFI, Monte FJQ, Villar JDF 2002. A new strycnobrasiline base of Strychnos mattogrossensis. Nat Prod Lett 16: 229-223.

Bettolo M, Battista G, Galeffi C, Nicoletti M, Messana I 1980. Alkaloids of Strychnos rubiginosa. Phytochemistry 19: 992-994.

Biocaa E 1954. Pesquisa sobre o método de preparação do curare pelos índios. Revista do Museu Paulista N.S 8: $164-226$

Bisset NG 1970. The African species of Strychnos. Part I. The ethnobotany. Lloydia 33: 201-243.

Bisset NG, Phillipson JD 1971. The African species of Strychnos. Part II. The alkaloids. Lloydia 34: 1-60.

Bisset NG 1974. The Asian species of Strychnos. Part III. The ethnobotany. Lloydia 37: 62-107.

Bisset NG, Phillipson JD 1976. The Asian species of Strychnos. Part IV. The alkaloids. Lloydia 39: 363-325.

Bruneton J 1999. Monoterpenoid indole alkaloids. In: Pharmacognosy, phytochemistry and medical plant. 2 ed. France: Lavoisier Publishing, p. 1000-1043.

De Medeiros CLC, Thomas G, Mukherjee R 1991. The source of $\mathrm{Ca}^{2+}$ for the spasmolytic actions of longicaudatine, a bisindole alkaloid isolated from Strychnos trinervis. Phytother Res 5: 24-28.

Delle-Manache F, Aldo PT, Bettolo GBM 1969. Occurrence of nor-dihydrotoxiferine in Strychnos pseudoquina St. Hil. Tetrahedron Lett 25: 2009-2012.

Ducke A 1965. O gênero Strychnos no Brasil. Boletim Técnico do Instituto Agronômico do Norte 30: 1-64.

European Office of Patent: banco de dados. http://www.inpi.gov. br/sites/links.htm, acessada em 04 março de 2004.
Frederich M, Depauw MC, Llabres G, Tits M, Hayette MP, Brandt V, Penelle J, Demol P, Angenot L 2000. New antimalarial and cytotoxic sungucine derivatives from Strychnos icaja roots. Planta Med 66: 262-269.

Forgacs P, Jehanno A, Provost J, Thal C, Guilhem J, Pascard C, Moretti C 1986. An indole alkaloid from Strychnos erichsonii. Phytochemistry 25: 969-971.

Galeffi C, Marini-Bettolo GB 1981. On the alkaloids of Strychnos-XXXVI. Tetrahedron 37: 3167-3170.

Galeffi C, Patamia M, Nicoletti M, Messana I, Marini-Bettolo GB 1982. 3-hydroxydiaboline, a tertiary alkaloid from Strychnos castelnaeana. Phytochemistry 21: 2393-2395.

Iwataki I, Comin J 1971. Studies on Argentine plants-XXXI. Tetrahedron 27: 2541-2552.

Juliem LD 1999. Fruit consumption, seed dispersal and fate in the vine Strychnos erichsonii in a Freanch Guianan forest. Revue d'Ecologie la Terre et la Vie 54: 315326.

Lorenzi H 1998. Árvores brasileiras: Manual de identificação e cultivo de plantas arbóreas nativas do Brasil. 2.ed. Nova Odessa: Plantarum.

Manga MH, Quetin-Leclercq J, Llabres G, Belem PML, Arnaldo F, Rocha I, Angenot L 1996. 9-Methoxygeissoschizol, an alkaloid from bark of Strychnos guianensis. Phytochemistry 43: 1125-1127.

Mukherjee R, Melo MDF, Santos CAM, Das BC, Guittet E 1990. Trinervine, a new indole alkaloid from Strychnos trinervis. Heterocycles 31: 1819-1822.

Mukherjee R, Silva BA, Das BC, Keifer PA, Shoolery JN 1991. Structure and strereochemistry of divaricine, a new bisindole alkaloid from Strychnos divaricans. Heterocycles 32: 985-990.

Mukherjee R, Das BC, Keifer PA, Shoolery JN 1994. Structure and stereochemistry of divarine another new bisindole alkaloid from Strychnos divaricans. Heterocycles 38: 1965-1970.

Mukherjje R, Silva TMS, Guimarães JBL, Oliveira ED, Keifer PA, Shoolery JN 1997. Tertiary alkaloid fraction of Strychnos atlantica: Confirmation of the identity and structures of indole alkaloids by high field nuclear magnetic resonsnce spectroscopy. Phytochem Anal 8: 115-119.

Mukherjee R, Keifer PA 2003. Warifteine and methylwarifteine: ${ }^{1} \mathrm{H}$ and ${ }^{13} \mathrm{C}$ assigments by two dimensional NMR spectroscopy. Org Magn Reson 41: 213-218.

Nicoleti M, Goulart MOF, Delima RA, Goulart AE, DelleMonache F, Bettolo GBM 1984. Flavonoids and 
alkaloids from Strychnos pseudoquina. J Nat Prod 47: 953-957.

Ohiri FC, Verpoorte R, Baerheim SA 1983. The African Strychnos species and their alkaloids: A review. $J$ Ethnopharmacol 9: 167-223.

Oliveira EJ, Medeiros IA, Mukherjje R 1996. Hypotensive and spasmolytic effects of normascusine B from Strychnos atlantica root. Phytomedicine 3: 45-49.

Penelle J, Monique T, Philippe C, Viviane B, Michel F, Angenot L 1999. Guiaflavine a new bisindole quaternary alkaloid from the stem bark of Strychnos guianensis. J Nat Prod 62: 898-900.

Penelle J, Monique T, Philippe C, Viviane B, Michel F, Angenot L, Jordi M 2000. Quaternary indole alkaloids from the bark of Strychnos guianensis. Phytochemistry 53: 1057-1066.

Penelle J, Monique T, Philippe C, Viviane B, Michel F, Angenot L, Jordi M 2001. 5',6'-Dehydroguiachrysine and 5',6'-dehydroguiaflavine, two curarizing quaternary indole alkaloids from the stem bark of Strychnos guianensis. Phytochemistry 58: 619-626.

Pinheiro MLB 2000. Contribuição ao estudo fitoqúimico do gênero Strychnos da flora Amazônica. Fortaleza, 260p. Tese de Doutorado em Química Orgânica Universidade Federal do Ceará, UFC.

Phillipe G, Angenot L, Monique T, Michel F 2004. About the toxicity of some Strychnos species and their alkaloids. Toxicon 44: 405-416.

Silva TMS, Silva BA, Mukherjje R 1999. The monoterpene alkaloid cantleyine from Strychnos trinervis root and its spasmolytic properties. Phytomedicine 6: 169176.

Silva BA, Dearaujo AP, Mukherjee R, Chiappeta AD 1993. Bisnordihydrotoxiferine and vellosimine from Strychnos divaricans root spamolytic properties of bisnordihydrotoxiferine. Phytother Res 7: 419-424.

Thomas G, Diniz MFFM, Mukherjje R 1992. Further studies on the antidiarrheal activity of binordihydrotoxiferine, a tertiary indole alkaloid in rodents. Phytother Res 6: 84-88.

Verpoorte R, Hylands PJ, Bisset NG 1997. Carbon-13 NMR spectroscopy of some Strychnos alkaloids. Org Magn Reson 9: 567-571. 